\title{
Buenas prácticas en la dirección de trabajos de fin de grado. Especial referencia al área jurídica
}

Good practices in graduation dissertations monitoring. Special reference to the legal area

\author{
Antonio Legerén Molina \\ Profesor contratado \\ Doctor en Derecho civil \\ Universidade da Coruña \\ Email: antonio.legeren@udc.es
}

Resumen: El objetivo de este trabajo es ofrecer orientaciones y modos prácticos de abordar la tarea de tutorizar la elaboración del Trabajo de Fin de Grado, culmen del proceso formativo de cualquier estudiante. Las reflexiones que se recogen en este texto son fruto de las opiniones de los estudiantes tutorizados, así como de la dirección a lo largo de los últimos años de más de medio centenar de TFG, y, aunque resultan útiles en general para cualquier tipo de Trabajo de Fin de Grado, tienen especial utilidad para los del área jurídica. Las buenas prácticas que aquí se desarrollan pretenden colmar buena parte de los problemas manifestados por los estudiantes y se agrupan en torno a tres bloques diferenciados: las relativas a la reunión inicial entre el tutor y el estudiante, las que conciernen a la operativa de la labor tutelar durante la confección del TFG y las atinentes a la fase que tiene lugar tras su conclusión.

Palabras clave: Buenas prácticas; tutorización del Trabajo de Fin de Grado; acompañamiento; autonomía. 


\begin{abstract}
This work aims to offer guidance and practical ways to approach the task of tutoring the Graduation Dissertation which is the culmination of the learning process of every student. The reflections contained in this text are the result of the opinions of the tutored students, as well as of the supervisory task of more than fifty Graduation Dissertations over the last few years, and although they might be helpful in general for any type of Dissertations, they are especially useful for those in the legal area. The good practices developed here are intended to address many of the problems expressed by students themselves and are grouped around three different blocks: those relating to the initial meeting between the tutor and the student, those concerning the operation of the monitoring work during the preparation of the Graduation Dissertation, and those relating to the phase that takes place after its conclusion.
\end{abstract}

Keywords: Good practices; supervision of Graduation Dissertation; accompaniment; autonomy.

\title{
1. Introducción y metodología
}

En ocasiones se ha dicho que el Trabajo de Fin de Grado es el culmen del proceso formativo de cualquier estudiante pues en él se han de compendiar y mostrar las actitudes, conocimientos, habilidades y competencias adquiridas a lo largo del Grado ${ }^{1}$. Sea ello así o

\footnotetext{
${ }^{1}$ De acuerdo con lo que señalan VALDERRAMA ET ALII $(2010,107)$, en lo que ahora importa, con el Trabajo de Fin de Grado se pretende que el estudiante muestre un "dominio integrado" de las competencias que ha venido desarrollando durante el Grado y/o que complete tal desarrollo. En efecto, según dispone el Real Decreto 1393/2007, de 29 de octubre, por el que se establece la ordenación de las enseñanzas universitarias oficiales (BOE $\mathrm{n}^{\circ} 260$, de 30 de octubre de 2007, modificado por el Real Decreto 861/2010, de 2 de julio, BOE $n^{\circ} 161$, de 3 de julio de 2010), el Trabajo de Fin de Grado deberá tener entre 6 y 30 créditos y estar "orientado a la evaluación de competencias asociadas al título" (art. 12.7 RD). La amplitud recogida en el citado Real Decreto ha de ser concretada en cada Universidad y en cada Facultad, a fin de diseñar un Trabajo de Fin de Grado que, reuniendo los requisitos impuestos por la norma, se ajuste a las necesidades particulares, impulsando y fomentando las destrezas propias de cada Grado. Son, por tanto, los Proyectos de Grado de cada titulación los que señalan las competencias específicas de dicho Trabajo. A modo de ejemplo, en el Reglamento del Trabajo de Fin de Grado en Derecho de la Universidade da Coruña (UDC) modificado por la Comisión de Docencia el día 3 de mayo de 2013 y el día 13 de septiembre de 2019-, se indica en el apartado 2.1 que "en este trabajo deben integrarse y desarrollarse los contenidos formativos recibidos, así como las capacidades, competencias y habilidades adquiridas durante el período de docencia del Grado". Por su parte, el artículo 2.2 apunta que "el TFG deberá estar orientado a aplicar las competencias generales asociadas al título de graduado en Derecho. En concreto, a capacitar para la búsqueda, gestión, organización e interpretación de datos relevantes con el objeto de emitir juicios que incluyan una reflexión jurídica sobre temas relevantes de la titulación de índole social, científica, tecnológica o ética, y facilitar el desarrollo de un pensamiento y juicio crítico, lógico y creativo". Por otra
} 
no en la práctica, es innegable que se trata de un trabajo con un peso relevante en la formación del alumnado.

La experiencia en la dirección de TFG muestra que, al menos en el ámbito jurídico, los estudiantes se encuentran con ciertas dificultades para su elaboración. Los problemas más comúnmente señalados por ellos mismos en las entrevistas personales realizadas suelen ser los siguientes: desconocimiento de las habilidades de cita y escritura académica, falta de seguridad en lo que al manejo de las fuentes de investigación se refiere, deficiencias en el proceso de expresión y, sobre todo, el hecho de ser el TFG la primera vez que abordan un trabajo de tal calado. En efecto, en buena parte de los casos constituye la primera ocasión en que los estudiantes se enfrentan a la redacción de un texto de cierta longitud y para el que, con más frecuencia de la deseada, suelen manifestar que "nadie les ha enseñado cómo proceder".

No compete aquí resolver cómo ha de colmarse la, en su caso, pretendida ausencia de formación ni enjuiciar si las pautas que actualmente se proporcionan, la bibliografía recomendada sobre la materia, o los talleres explicativos que se suelen desarrollar en las diversas Facultades son suficientes o no ${ }^{2}$. Tal es el objeto de otros estudios ${ }^{3}$. El presente texto solo pretende dar respuesta a buena parte de los problemas que suelen experimentar los estudiantes - tal y como ellos mismos señalan- pero desde la perspectiva del otro

parte, se advierte ya desde ahora que las referencias a "los docentes", "los estudiantes" o "los alumnos"-en masculino- incluyen obviamente a las personas de ambos sexos. Se ha optado por tal modo de proceder para evitar recargar la escritura.

2 A modo de ejemplo, en la Facultad de Derecho de la UDC se facilita a los alumnos una amplia información, tanto del proceso de selección de temas para el TFG, nombramiento de tutores u otras cuestiones procedimentales a fin de que sepan qué paso dar en cada momento, como de modos de cita y escritura -las llamadas "Pautas de estilo" para la elaboración del TFG-. La experiencia muestra que tal información resulta de notable utilidad al estudiante.

${ }^{3}$ Ante la extensa bibliografía existente relativa a los trabajos con que finalizan el Grado o el Posgrado, a continuación únicamente se ofrecen algunas de las referencias que se consideran más interesantes sobre materias distintas de la que se analiza en este estudio. Así, en relación con los diversos aspectos del proceso de elaboración y tutorización de tales trabajos -temporalización, desarrollo, resultados de aprendizaje, etc.vid., entre otros, DE PRO, SÁNCHEZ y VALCÁRCEL, 2013, 728-748 y TUR-VIÑES ET ALII, 2013, 86-104 ambos referidos específicamente a TFM- o TARÍ ET ALII, 2015, 1357-1375. Respecto de la evaluación de los TFG, vid. BONILLA y MARTÍN, 2012, 241-253 y, sobre la evaluación, más en general, VALLÉS, UREÑA y RUIZ, 2011, 135-158. Finalmente, sobre algunos aspectos particulares -por ejemplo, líneas temáticas, elementos éticos relacionados con la cooperación y el desarrollo, proyectos de aprendizaje-servicio, entre otros-, vid. las referencias contenidas en DíAZ-VÁZQUEZ ET ALII, 2018, 161. 
término de la relación tutelar que se crea en los TFG: el tutor. Es decir, lo que se pretende en las páginas que siguen es poner por escrito algunas ideas y buenas prácticas con el fin de resolver las dificultades comúnmente sentidas por los alumnos de TFG y que son fruto de lo por ellos manifestado y de la dirección a lo largo de los últimos años de más de medio centenar de TFG. Por ello, tampoco se quiere abordar ahora si las herramientas de que actualmente dispone el docente son adecuadas o no para la tutela de los TFG o si se ha recibido suficiente formación específica para tal tarea ${ }^{4}$. El propósito que se persigue es, según se ha dicho, ofrecer orientaciones y modos prácticos de abordar la tarea de tutorizar a los estudiantes en la elaboración del TFG, por si acaso resultasen útiles a algún docente en una situación parecida ${ }^{5}$. Aun cuando el objetivo apuntado no sea el más ambicioso posible, no son pocas las dificultades que entraña elaborar un texto de este estilo pues los tipos de trabajo que se suelen realizar como culmen del Grado pueden ser -y sonextraordinariamente variados ya que cada rama de conocimiento tiene su propia fisonomía y peculiaridades. De todas maneras, considero que las reflexiones que a continuación se

\footnotetext{
${ }^{4}$ Destacando ya desde los primeros compases del proyecto de creación del Espacio Europeo de Educación Superior (EEES) que la formación del profesorado constituía una de las claves del triunfo de dicho Espacio educativo, vid. VALCÁRCEL, 2003 y GONZÁLEZ, 2005, 44, quien señalaba también algunas implicaciones concretas. Por otra parte, aun cuando comúnmente se reconoce el importante papel del tutor en la elaboración del TFG, su misión no está claramente definida ni desde una perspectiva teórica ni práctica (cfr. Río, DíAZ-VÁZQueZ y MASIDE, 2017, 159-172), existiendo, todo lo más, listas de roles que ha de desempeñar o clasificaciones de los distintos estilos de tutorización (cfr. DíAZ-VÁZQUEZ ET ALII, 2018, 167 y ss. y la doctrina que allí se cita). Con todo, la conciencia de la referida importancia es una de las razones que lleva a los Centros Universitarios para la Formación e Innovación Educativa que, con este u otros nombres, existen en las distintas Universidades a ofrecer cursos para mejorar la tutorización de TFG. En efecto, aun cuando hay ciertos elementos en el desempeño de la relación tutelar que se adquieren por la experiencia, el papel insustituible del tutor demanda formación específica para llevarlo a cabo. En tal sentido, para ejercer la labor tutelar no basta con poseer un elevado nivel de conocimientos académicos, siendo necesario desarrollar habilidades de carácter interpersonal o relacional. En lo que hace a estas últimas, MARTín y NiEVA $(2016,16)$ apuntan que "la tarea de tutela del TFG implica un papel multidimensional en el que hay que poner en juego habilidades de guía, de crítica intelectual y de apoyo, además de tener un conocimiento declarativo sobre cómo realizar este tipo de trabajos". Así las cosas, cabe señalar que junto con el tutor - principal apoyo del estudiante y cuya tarea se ha vinculado en cierta medida al éxito o fracaso del TFG (cfr. HeInZE y HeinZe, 2009, 294-305 y MolinA, RodríGueZ y Colmenero, 2020, 241-250) - también se han desarrollado estudios que analizan la denominada "tutoría por pares". A modo de ejemplo, vid. SIOTA, 2015, 1-18.
}

5 En este contexto, no está de más destacar que la Ley Orgánica 6/2001, de 21 de diciembre, de Universidades configura el "asesoramiento y asistencia" universitaria -en sentido amplio; es decir, no referida de manera específica o particular a la que es objeto de análisis en este trabajo- como un derecho (cfr. art. 46.2.e). En sentido parecido se contempla en los artículos 7 a 10 del Real Decreto 1791/2010, de 30 de diciembre, por el que se aprueba el Estatuto del Estudiante. Vid. también los artículos 19 a 21 del mismo texto normativo que recogen principios generales aplicables a las tutorías así como las diversas clases de ésta. 
efectúan resultan útiles en general para todos ellos, y de modo particular para los supuestos en que los mencionados trabajos consistan en una tarea de investigación o en la elaboración de un dictamen o resolución de un caso práctico -elementos típicos de los estudios de Derecho- ${ }^{6}$.

La metodología utilizada para la obtención de la información e ideas contenidas en este texto ha consistido en la recolección, por medio de entrevistas personales, de las opiniones de los alumnos tutorizados por parte del autor desde 2013 hasta la actualidad. En ellas, y de acuerdo con un esquema prefijado, se abordaban cuestiones procedimentales, metodológicas, sustantivas y actitudinales en la elaboración del $\mathrm{TFG}^{7}$. Igualmente, tal análisis se ha enriquecido con las experiencias consideradas más útiles en esta labor aportadas por diversos tutores. Ello expuesto, las buenas prácticas que aquí se desarrollan se agruparán en tres bloques: las relativas a la reunión inicial entre el tutor y el alumno, las que conciernen a la operativa de la labor tutelar durante la confección del TFG y las atinentes a la fase que tiene lugar tras su conclusión ${ }^{8}$.

\footnotetext{
${ }^{6}$ Aunque el título de este trabajo circunscriba el contenido de estas páginas al TFG, lo que a continuación se señala resulta aplicable en gran medida a los Trabajos de Fin de Máster. Ello, aun cuando entre ambos tipos de trabajo medien notables diferencias relativas tanto a los requisitos intelectuales que exigen, los calendarios de realización, el número de estudiantes que los desarrollan y el tiempo que le dedican, la ratio tutor y tutelado, las necesidades de aprendizaje, etc... lo que ha permitido establecer una distinción entre la "supervisión" - propia del nivel de Posgrado-y el "asesoramiento" (advising) - propio del Grado- (HEALEY ET ALII, 2013, 59). Sobre estas cuestiones, vid. también DíAZ-VÁZQUEZ ET ALII, 2018, 161.

${ }^{7}$ El esquema a que se alude en el texto -cada bloque está integrado por diversas preguntas- se ha ido matizando y perfeccionando con el tiempo. La ausencia de una estructura invariada desde el inicio dificulta, a mi juicio, hacer análisis cuantitativos comparativos pues requerirían numerosas precisiones o matizaciones. Por tal motivo en este trabajo se ha prescindido de los datos numéricos, centrándose en los elementos y dificultades señaladas de manera mayoritaria por los estudiantes,

${ }^{8}$ La división señalada en el texto coindice sustancialmente con las tareas esenciales de la dirección académica de un TFG. A modo de ejemplo, en el ya citado Reglamento del Trabajo Fin de Grado en Derecho de la Universidade da Coruña, se señala en el artículo 3.1 que el profesor-tutor será responsable de “a) exponerle al / a la estudiante cuya tutoría ejerza las características del TFG, y orientarle/a en su desarrollo; b) realizar un seguimiento de la elaboración del TFG y velar por el cumplimiento de los objetivos fijados; c) autorizar el depósito y la defensa del TFG'. Con todo, no ha de olvidarse que, en la doctrina, se han ofrecido clasificaciones diversas tanto de las etapas del proceso de tutorización -más ajustadas a la modalidad de TFG o TFM de que en cada caso se trate-, como de los distintos tipos de tutoría -según el agrupamiento, la temática o el canal de comunicación- (RODRíGUEZ ET ALII, 2013, 40 y 45 a 47).
} 


\section{La reunión inicial}

Las técnicas de desarrollo de la memoria suelen señalar que una de las mejores formas de retener conceptos es por medio de su asociación con imágenes. Aplicando dicha regla al caso presente se puede afirmar que una figura que compendia muy bien la labor del tutor durante la elaboración del TFG es la de ser como la "torre de control" de un aeropuerto9. Los pilotos -en este caso, los estudiantes- son quienes han de llevar a buen término al pasaje $\mathrm{y}$, evidentemente, son sus conocimientos y destrezas las que permiten cumplir con la función o tarea encomendada. Ahora bien, en el desarrollo de dicha función, como es obvio, resultan de notable utilidad las directrices que emite la "torre de control" -la pista en que tomar tierra, la fuerza y orientación del viento, el estado del firme, etc.-. Pues bien, si se aplica esa metáfora en la elaboración de un TFG se consigue un doble efecto positivo: de una parte, se responsabiliza a los "pilotos" de que ellos -y solo ellos- son quienes pueden llevar a buen fin la tarea; reconocimiento o atribución de responsabilidad que suele producir un notable crecimiento personal en los estudiantes pues comprueban que son ellos los que han de enfrentarse con las dificultades que encontrarán durante la tarea, para cuya superación cuentan -eso sí- con el constante aliento y consejo de la "torre de control" ${ }^{10}$. Y de otra, la puesta en práctica de la referida imagen incide en el

\footnotetext{
${ }^{9}$ En la literatura relativa a los TFG se acude usualmente a la figura del andamiaje o scaffolding, acuñada por WOOD, BRUNER y ROSS (1976, 90), recurriendo a la metáfora de "apoyo a la construcción del conocimiento" de los tutelados: "more often tan not, it involves a kind of scaffolding process that enables a child or novice to solve a problem, carry out a task or achieve a goal which would be beyond his unassited efforts. This scaffolding consists essentially of the adult "controlling" those elements of the task that are initially beyond the learner's capacity, thus permitting him to concentrate upon and complete only those elements that are within his range of competence". Tales autores analizan con más detalle el proceso de "scaffolding" en las pp. 98-99. En nuestro país, también se ha señalado que la tutoría es un proceso de acompañamiento, guía o asistencia (GARCíA ET ALII, 2005, 191) y para representar el rol orientador aunque en el caso que se cita, referido a la de carácter laboral y no específicamente a la tutoría- se ha recurrido a la metáfora de la persona que ayuda a otra a montar en bicicleta (ALTUNA, 2005, 109-114). Según se advierte, los referidos conceptos, aplicados a lo que ahora interesa, se refieren exclusivamente en la tarea realizada por un docente y no a la tutoría hecha por pares.

10 "El papel del tutor o tutora es el de servir de guía y apoyo en este proceso, ofreciendo las herramientas adecuadas para salvar los obstáculos que el estudiante encontrará en su camino" (MARTÍN y NIEVA, 2016, 18). Ello es así, aun cuando como se indica en el texto, la principal responsabilidad así como la iniciativa corresponde al estudiante (en el mismo sentido, ibídem, p. 18 donde también se menciona que la motivación del estudiante "debe ser intrínseca y no basarse en la aplicación de reglas y mandatos estrictos por parte del tutor").
} 
sentido de servicio de la "torre de control": ha de facilitar la orientación y estímulo necesario para que el "piloto" culmine con éxito su misión"11.

Según muestran los resultados de las entrevistas personales realizadas, las ideas señaladas han de conformar uno de los principales contenidos que han de ser explicados en la reunión inicial con los alumnos, previa a la elaboración del TFG. Cuando sean varios los estudiantes que vayan a ser tutorizados por una misma "torre de control" resulta oportuno realizar una reunión conjunta con ellos. De esta manera reciben una misma información, se ahorra tiempo y, finalmente -y sobre todo- les permite advertir a nivel individual que no son los únicos que han de realizar tal tarea: ni están solos en el desempeño de tal función -habitualmente comprueban que todos se encuentran en una misma situación de “desconocimiento de por dónde avanzar"- ni son los únicos - por lo que advierten la necesidad de cierto orden o sistema en la elaboración del TFG y la correspondiente tutorización-12.

Junto con la explicación de la misión de cada una de las partes implicadas en la relación tutelar, resulta de notable ayuda la contextualización del TFG en el proceso formativo del alumno $^{13}$. Por lo menos, y tal y como han manifestado en las conversaciones mantenidas para la confección de este estudio, así lo perciben ellos. No se trata, en este punto, de elaborar interminables discursos sobre el valor que para el futuro de cada estudiante tendrá la realización del trabajo, apelando, en consecuencia, a su responsabilidad, sino de, brevemente, hacerles ver el papel que tiene una excelente elaboración del TFG en el

\footnotetext{
${ }^{11}$ Tal estímulo y apoyo resulta un elemento necesario para la creación del, denominado por BAIN (2007, 29 y 114 y ss.), “entorno para el aprendizaje crítico natural”, imprescindible para la consecución del objetivo que se pretende.
}

12 Además de por lo expuesto en el texto, tales reuniones grupales ayudan al estudiante a compartir inquietudes, comentar problemas procedimentales que pueden concurrir en varios o le facilita ser consciente de que sus dificultades u obstáculos son compartidos por otros. En este sentido, vid. RoDríGUEZ, 2004, 99-123 y MARTín y NIEVA, 2016, 20.

13 Constituye una cuestión debatida la oportunidad de redactar por escrito un "acuerdo" que explicite el contenido y "obligaciones" de cada una de las partes de la relación tutelar, y que han de firmar ambas. A mi juicio, mala sería la situación si hubiese de recurrirse a la formalización de tal acuerdo que, en un contexto normal, existe pero de carácter verbal. En mi opinión, únicamente podría resultar útil redactar tal acuerdo si ayuda al estudiante a la concienciación sobre la tarea a desempeñar. De todos modos, como se indica, se considera preferible que sea verbal. A favor de su escritura, vid., entre otros, INGELLIS y PICAZO, 2015, 542 y ss. 
desempeño de su labor profesional ${ }^{14}$. En este sentido, la experiencia muestra también la utilidad de explicar a los estudiantes que la labor de la "torre de control" en este caso no es primariamente ayudarles a superar una específica prueba sino "darles una mente", ayudarles a que adquieran unas habilidades y destrezas relativas al proceso intelectual que conlleva el TFG, así como mejorar su modo de redactar -aspecto que ordinariamente pondrán en juego de forma continuada en su quehacer profesional, de manera especial cuando se trate de estudiantes de Derecho- ${ }^{15}$. Asimismo, no está de más apuntar que, probablemente, en el futuro no tendrán la posibilidad de disponer de un tutor ni de tanto tiempo dedicado específicamente a mejorar los fundamentos y destrezas necesarias para desarrollar su trabajo, lo que les proporcionará la seguridad que pretenden y cuya ausencia suelen acusar, al menos, en el momento inicial del TFG.

En el contexto apuntado se ha revelado útil la utilización de una "herramienta tutelar" a modo de mapa o guía que facilite y contextualice las diversas tareas necesarias para la realización del trabajo ${ }^{16}$. Aunque el número de alumnos/as comprendidos en este estudio que ha usado dicho instrumento es pequeño -6 de $71-$, los que lo han utilizado han mostrado gran satisfacción. Evidentemente, aun cuando el contenido de dicha herramienta

\footnotetext{
${ }^{14}$ En tal contexto, es oportuno que el tutor señale a los estudiantes que la elaboración del Trabajo de Fin de Grado es una ocasión singular para desarrollar destrezas que, muy posiblemente, hasta ahora no habían trabajado, siendo una de ellas la metacognición. El tutor debe ayudar o promover el referido metaconocimiento del propio estudiante, ya en su vertiente declarativa -las competencias y flaquezas que cada uno tiene para poner en marcha el trabajo, así como las características y estrategias a seguir en su desempeño-, ya en la procedimental -el control del uso de tales conocimientos y de los procesos que se activan al realizar la tarea encomendada-; en otros términos: el centrado en el "saber" cómo es uno y lo que ha de realizar, y el orientado al "saber hacer" que se despliega al actuar. Sobre ambos tipos de metacognición vid. POZO y MATEOS, 2009, 54-69, y, sobre algunos modos de concretarlos y estimularlos vid. MARTÍN y NIEVA, 2016, 32.
}

${ }^{15}$ De acuerdo con lo señalado en el texto, un elemento fundamental de la relación tutelar es la promoción de la autonomía en el estudiante (sobre la importancia de la autonomía y la oportunidad que el tipo de trabajos que se comentan suponen para su consecución vid. TODD, SMITH y BANNISTER, 2006, 161 y ss.). Así, el objetivo fundamental del TFG no es tanto la elaboración de un buen trabajo cuanto que ese buen resultado sea porque se haya adquirido la referida autonomía. En efecto, en atención a que lo mejor del trabajo "queda en el estudiante", parece oportuno hacérselo notar, pues le servirá de motivación cuando vengan momentos de menor ánimo con la tarea a realizar. Si el estudiante adquiere tales habilidades sabrá enfrentarse a las nuevas situaciones que la vida profesional le deparará, aprovechándolas como ocasiones para el aprendizaje y el desarrollo de nuevas destrezas. Así las cosas, la consecución de tal autonomía es un trabajo laborioso que dista mucho de que el tutor "abandone" al estudiante a su suerte: supone mantener "cercanía en la distancia" a la vez que se le proporcionan las herramientas necesarias -explicándole su modo de uso-.

${ }^{16} \mathrm{Cfr}$. Anexo I donde se incluye un ejemplo de herramienta tutelar. 
habrá de adaptarse al tipo de Grado y a las características del TFG de que en cada caso se trate, lo que se pretende con ella es ayudar al estudiante a que reflexione antes, durante y después de la realización del trabajo: que sepa los porqués y, sobre todo, se fije los adónde quiere llegar con el $\mathrm{TFG}^{17}$. De manera secundaria y natural, el uso de la herramienta establece cierto sistema y, por decirlo de algún modo, calendariza la tarea a realizar. En efecto, el recurso a este instrumento permite pedir a los estudiantes que presenten un calendario de trabajo para poder coordinar la labor de tutorización de todos. Y la experiencia muestra que solo el hecho de que plasmen por escrito dicho calendario con las tareas a desempeñar constituye una ayuda reflexiva sobre el trabajo que les permite "poner los pies en el suelo" sobre el tiempo disponible para cada una de las labores que implica el TFG -búsqueda de información, lecturas detenidas, etc.- ${ }^{18}$, así como conocer en cada momento cuál es la siguiente tarea o el siguiente paso a realizar, anticipándose de esa manera a lo que está por venir. Asimismo, este modo de proceder facilita que no se acumulen revisiones de textos cerca del plazo límite de entrega que impiden un desempeño y tutorización excelente, que es lo que se pretende.

A mi juicio, y a la vista de lo manifestado por los alumnos que han formado parte de este estudio, dos cuestiones más han de abordarse en dicha reunión inicial con los estudiantes: de una parte, la explicación de la operativa de la labor de tutoría; de otra, facilitar un conjunto de reglas formales sobre el modo de redactar.

En cuanto a la operativa, la experiencia enseña que es conveniente no esperar para comenzar la corrección a que el estudiante haya elaborado el trabajo en su totalidad. Así, en primer término, un paso que el alumno ha de dar cuanto antes es la elaboración del índice y su presentación para revisión. Evidentemente, resulta inusual que el inicialmente elaborado se mantenga estático y permanezca invariado en el tiempo. Pero ello no resulta en absoluto problemático pues, nuevamente, la plasmación del índice: concede realismo al trabajo a desarrollar, permite detectar, si fuese el caso, algún error notable que pudiese

\footnotetext{
${ }^{17}$ En conexión con lo señalado en el texto, vid. el recurso a la Guía del estudiante que explican MARTÍN y NIEVA (2016, 33-34) como herramienta para favorecer el metaconocimiento declarativo.

${ }^{18}$ Vid. el amplio listado de tareas a desarrollar por el alumno (reuniones con el tutor, asistencia a reuniones formativas, revisión bibliográfica, lectura de los trabajos encontrados, planteamiento de hipótesis y diseño de la investigación, etc.) que se encuentra en MARTín y NIEVA, 2016, 9. A lo que parece, el recurso a tal lista de tareas resulta especialmente útil cuando se les asigna una estimación de las horas que supondrá cada una pues estimulará el metaconocimiento declarativo al que con anterioridad se ha hecho referencia.
} 
desviar el trabajo de los fines pretendidos, y, finalmente, propicia que los cambios que ulteriormente introducen en él los alumnos sean fruto de una labor reflexiva.

De acuerdo con el modo de proceder indicado, y una vez se les anime a comenzar la redacción a fin de poder ir dándoles orientaciones precisas sobre ella, es oportuno pedir a los estudiantes que entreguen el primer apartado -capítulo, pregunta, sección, según los casos- para su corrección una vez lo terminen. Eso sí, solamente han de enviarlo cuando piensen que está totalmente finalizado - “visto para sentencia"-, tanto desde un punto de vista formal como sustantivo. Este requerimiento evita improcedentes pérdidas de tiempo -que el alumno pueda señalar en una sesión presencial de corrección que "esto ya lo he modificado o suprimido en la nueva redacción"-, a la vez que, y esto es lo más importante, ayuda a transmitir la "mente" a que antes se ha aludido. En efecto, ordinariamente muchos de los errores o aspectos a mejorar que se encuentran en ese primer apartado se suelen repetir en los demás. Por ello, carece de sentido demorar el inicio de la corrección a tener el trabajo completamente terminado. Además de que la redacción de todo el trabajo retrasa su entrega -y, en consecuencia, se dispondrá de menos tiempo para su revisión y, en su caso, modificación-, se advertirán repetidos los errores o aspectos a mejorar, con la considerable carga de tiempo y esfuerzo que supone su cambio a lo largo de todo el trabajo. Se advierte que es más eficaz corregir las veces que haga falta el primer apartado hasta que se aprecie que el estudiante va "adquiriendo la mente" señalada. La adquisición de ese "modo de trabajar" hará que mejore tanto ese apartado corregido como la primera redacción del resto de capítulos, lo que redundará en un aumento de la velocidad de trabajo sin descuidar su calidad. Además se comprueba que la revisión del segundo y ulteriores capítulos suele resultar más ágil pues es el propio estudiante el que habrá eliminado por sí mismo los errores cometidos en el primero. De esta manera se consigue uno de los más altos objetivos de la labor de tutoría: que el estudiante aprenda de manera autónoma.

En mi opinión, también resulta conveniente elaborar y entregar en esta reunión un conjunto de reglas formales sobre el modo de escribir. La razón se puede resumir señalando que si no, habitualmente "los árboles no dejan ver el bosque": un trabajo con muchos errores formales impide al revisor llegar al fondo de lo que el estudiante quiere decir. En tal sentido, la labor de tutorización se presenta como una labor por capas, al 
modo de la jardinería: en primer lugar hay una fase de desbrozar y eliminar los elementos grandes, tras la cual se descubren en esa misma parcela nuevos elementos a ir puliendo que antes no se advertían pero que estaban ahí; además, suprimidos los elementos más voluminosos, se pueden calibrar de manera clara las dimensiones, profundidad y posibilidades del jardín. Las revisiones ulteriores serán, en consecuencia, para ir mejorando, hasta el detalle, el jardín que ahora es una realidad y antes solo se vislumbraba. Algo similar sucede con los TFG. La labor de "desbroce" consiste en respetar una serie de reglas formales, cuya ausencia impide acceder al fondo de lo que se quiere exponer. Por ello es muy oportuno facilitar tales reglas en esa reunión inicial señalando al estudiante que no se leerá el trabajo si no se cumplen: el respeto de las reglas constituye un presupuesto imprescindible ${ }^{19}$. Es más, si se advierte que el alumno entrega el texto sin cumplir tales requisitos, entiendo que lo oportuno es devolvérselo sin leerlo de manera completa pero señalando qué cambios ha de realizar. No se trata de marcar todas las manifestaciones de un mismo error sino de señalar el error mismo para que sea el estudiante el que se ejercite y descubra de manera autónoma tales faltas y las subsane; lo que le irá dando la "mente" mencionada. Este modo de proceder supondrá una ayuda inicial para que desarrollen destrezas en la escritura, en la que -como suelen exponer- habitualmente no gozan de mucha experiencia y cuya incorporación supondrá un esfuerzo suplementario tanto por su parte como por la del tutor. El cuidado de la

\footnotetext{
${ }^{19}$ Para la elaboración de las reglas formales mencionadas en el texto resulta eficaz su agrupación en categorías. Así, respecto del índice cabe recomendar, entre otras cuestiones, que solo se ponga un apartado 1 si va a haber un 2 o que se revise que los títulos de los epígrafes que figuran en el texto coinciden totalmente -en fondo y en forma- con los que aparecen en el índice, o, en fin, que la elección de los títulos refleje fielmente el contenido de cada epígrafe. En relación a las abreviaturas cabe aconsejar, entre otras cosas, la oportunidad de valorar la conveniencia de dedicar una página a ellas o no, en función de si son muchas o pocas, comunes o inusuales, etc., así como que no se utilicen en el texto corrido. En lo que hace a la redacción, ha de revisarse que se usa un único tipo de letra y se eliminan los dobles espacios, o cuidar que las comillas de cierre vayan antes del número de nota y del punto y seguido o aparte. En el ámbito jurídico, parece oportuno que todas las alusiones a preceptos incluyan una referencia a la norma en que se integra o que se utilice tenor jurídico en la redacción, despersonalizando la escritura. Finalmente, respecto de las notas y el modo de cita, facilita la lectura del texto si no se ubican en mitad de una frase de modo que su lectura corte la del texto principal, ni antes de un número como si fuese una potencia matemática. Cuando se cite literalmente en el texto principal la idea de un autor no es preciso poner el "confróntese" (cfr.), a diferencia de cuando no se trata de una cita literal. Igualmente, han de fijarse criterios uniformes sobre el formato de los textos legales, la jurisprudencia y la doctrina de manera que permita su identificación. Y, por último, a modo de ejemplo, ha de recomendarse sentido común a la hora de citar: es tan erróneo e injusto no hacerlo cuando se debe, como citar para algo intrascendente y al alcance de cualquiera.
} 
forma, además de suponer una muestra de consideración hacia el potencial lector del trabajo, da mayor brillo y lustre al contenido, a la vez que destaca una actitud de cuidado de los detalles que resulta extraordinariamente positiva para el desarrollo de cualquier labor profesional ${ }^{20}$. En este sentido, no son pocas las Universidades que facilitan reglas sobre los modos de cita $\mathrm{u}$ otros elementos formales a fin de ayudar a los estudiantes a superar otra de las dificultades que, como se ha señalado páginas atrás, comúnmente señalan $^{21}$. Ahora bien, como tales pautas no suelen descender ni abarcar todos los detalles, considero conveniente la elaboración de un conjunto o decálogo propio que complemente el contenido de dichas instrucciones ${ }^{22}$.

${ }^{20}$ A mi juicio, resulta beneficioso ser exigente con las formas pues, según se apunta en el texto, es un
síntoma de profesionalidad. De ordinario resulta más sencillo que un trabajo formalmente cuidado aporte
buen contenido sustantivo que al revés. De todos modos, ha de advertirse que no se trata de ser rígido o
inflexible con los aspectos formales, sino que lo que se busca es desarrollar un espíritu de cuidado de los
detalles que, de adquirirse, afectará, lógicamente, tanto a la forma como al fondo. En tal sentido, una
recomendación práctica para el estudiante es que trabaje siempre con el formato definitivo de manera que
en las distintas revisiones podrá ir advirtiendo errores para ir corrigiéndolos. Entiendo que no es buena
estrategia "dejar las cuestiones formales para el final": puede que entonces no haya tiempo y, sobre todo,
impide adquirir esa forma de hacer a que se alude. Lo anterior resulta especialmente relevante con el modo
de cita de la bibliografía. La experiencia muestra que es más sencillo hacerlo bien desde el inicio, antes que
demorarlo para otro momento, pues, entonces, la labor de localizar las obras citadas resulta ardua y costosa.

${ }^{21}$ La fijación de las referidas pautas, aun cuando primariamente pudiese considerarse como una limitación de la autonomía del estudiante, en último término resulta algo beneficioso pues, además de introducirle en el lenguaje académico, homogeneizará los trabajos, lo que facilita su evaluación y comparación con otros (BATTANER, GONZÁLEZ y SÁNCHEZ, 2016, 51).

${ }^{22}$ Según se ha apuntado en el texto, en mi opinión, resulta oportuno complementar las "Pautas de estilo" que suelen ofrecer las Facultades con otras cuestiones más concretas a las que, obviamente, no ha de descender dicho documento. Un ejemplo de algunas recomendaciones específicas se contienen en la nota ${ }^{\circ}$ 19. Con tal modo de proceder se facilita al alumno la "alfabetización académica"; esto es, que conozca las reglas propias del estilo académico y de investigación, que habitualmente desconoce y que ordinariamente es de las competencias que inicialmente se les presenta como más dificultosa (sobre dicha "alfabetización" y cómo implementarla desde diversos prismas, vid. CARLINO, 2002, 43-61). Asimismo, también es la competencia que más se echa en falta desde la perspectiva de los tutores (cfr. MARTín, 2016, 149). Así pues, parece oportuno complementar las actuaciones de corte más institucional $-\mathrm{p}$. ej. a través de las asignaturas (cfr. CARLINO, 2002, 43-61) - con otras de índole más personal como la facilitación de las mencionadas pautas, la elaboración de plantillas o el feedback (en sentido parecido, INGELLIS y PICAZO, 2015, 547). Así las cosas, y tal y como se expone en el texto, la retroalimentación sobre el modo de escritura reviste particular importancia para la mejora del trabajo y, sobre todo, para la consolidación de destrezas en el estudiante (sobre esta cuestión vid. CANABAL y MARGALEF, 2017, 149-170). 


\section{La labor tutelar en el curso de la elaboración del trabajo}

Un elemento que resulta importante en el desarrollo de la labor tutelar mientras el estudiante elabora el TFG es no perder de vista el objetivo de dicha tarea: la adquisición de una serie de destrezas y de hábitos de razonamiento lógico. Es decir: que ejercite su proceso de razonamiento para llegar a unas conclusiones, y, respetando unas reglas formales, desarrolle su propio estilo de escritura. Tener presente esta finalidad impedirá configurar el estilo de los alumnos al modo propio, y demanda, por sí mismo, un tono siempre animante, donde se sugiera más que se imponga y se expliquen con delicadeza las razones y los porqués de tales sugerencias ${ }^{23}$. Asimismo, refrescar el objetivo señalado supondrá que el tutor habrá de desarrollar las acciones necesarias y dedicar el tiempo que en cada caso sea oportuno para conseguirlo. Y es que las necesidades y destrezas de cada estudiante son únicas y parcialmente distintas a las de los demás, motivo por el cual carece de sentido elaborar un procedimiento estándar e inflexible en la labor tutelar: el tutor ha de adecuarse al caso específico. Por ello, junto con la formación básica a que páginas atrás se aludía, hay otro conocimiento que solo se adquiere mediante su cultivo con personas diferentes y con necesidades diversas. De esta manera, la relación tutelar, antes que focalizada en el tutor, se centrará en el estudiante y en sus necesidades para alcanzar el objetivo señalado ${ }^{24}$.

Junto con el presupuesto anterior, hay otro que resulta de notable importancia: estimular en el estudiante un espíritu de "honradez intelectual". Se trata de que, desarrollando un hábito de razonamiento lógico, cada uno llegue a sus propias conclusiones, aun cuando para llegar a tal punto recurra de manera expresa y consciente a razonamientos, ideas o sugerencias de otros autores. Y, como adecuada manifestación de la justicia, es preciso

\footnotetext{
${ }^{23}$ Sobre la conveniencia de ofrecer feedback positivo o descriptivo, incidiendo en los elementos correctos antes que centrarse en los incorrectos, vid. RODRÍGUEZ ET ALII, 2013, 42.

${ }^{24}$ Lo expuesto en el texto no impide que existan algunos estándares comunes de necesidades que resulten similares en todos los estudiantes, tal y como se ha señalado páginas atrás y ha evidenciado la realización de este estudio. En cualquier caso, sí parece claro que el fomento de la autonomía, la delimitación del límite entre la parte emocional o intelectual en la labor tutelar o el modo de comunicación durante su desarrollo no podrán ser exactamente iguales en todo caso. Sobre estas cuestiones vid. MARTín y NIEVA, 2016, 17 y ss. En relación con la supervisión del estudiante, que no es un proceso estándar, vid. BECKMAN y HENSEL (2009, 40-44) quienes lo refieren de manera más general a la investigación de los alumnos de Grado. En efecto, la flexibilidad y capacidad de adaptación del tutor a las necesidades del tutelado son dos indicios de la calidad de la labor de supervisión.
} 
"dar a cada uno lo suyo", de modo que se han de citar con exactitud las fuentes a que se recurre de manera expresa o en las que uno se inspira ${ }^{25}$. Tal modo de proceder evitará plagios y, sobre todo, dará frescura al trabajo.

En cuanto a la operativa, los resultados del estudio realizado muestran la conveniencia de aplicar los tres elementos a que a continuación se alude. Primero: resulta interesante combinar sesiones presenciales con otras de carácter virtual. Aun cuando la herramienta de "control de cambios" de los programas para tratar textos resulten útiles para efectuar correcciones, por lo señalado por los alumnos se advierte la conveniencia de que algunas reuniones sean presenciales ${ }^{26}$. Esta presencialidad permite explicar mejor y en menos tiempo las razones de fondo de las sugerencias que se realizan, lo que ayudará a la adquisición de destrezas. Además, sirve a los alumnos para ver aplicadas las reglas de redacción elaboradas por la propia Universidad o por el propio docente, que es el mejor modo de incorporarlas al propio bagaje.

En segundo lugar ha de incidirse en que el trabajo ha de estar fundamentado: el TFG pretende dar respuesta a unos interrogantes o busca elaborar de manera solvente un tema. No se trata de "acarrear materiales" y/o, en el caso de los estudiantes de Derecho, citas doctrinales o jurisprudenciales, sino de "hacerlos propios" -citándolos oportunamente- de manera que puedan servir de respaldo a las opiniones que se mantienen ${ }^{27}$. Han de evitarse, por tanto, afirmaciones tajantes sin fundamento y consideraciones o discursos gratuitos que despisten del objetivo que se pretende. Asimismo, con un TFG no se trata de recopilar opiniones ajenas, sino, al hilo y con motivo de ellas, elaborar una opinión propia fundamentada y razonada ${ }^{28}$.

\footnotetext{
25 Vid., en tal sentido, MARín Vichis, 2017, 92. En efecto, no ha de perderse de vista que las lecturas realizadas son las que, inconscientemente, crean un humus que permite que germinen nuevas ideas que entonces resultan propias, personales.

${ }^{26}$ De la misma opinión son INGELLIS y PICAZO, 2015, 547.

${ }^{27}$ En palabras de SCARDAMALIA y BEREITER (1992, 43-48) se trata de evolucionar de la situación de "decir el conocimiento" a la de "transformar el conocimiento"; esto es, de la potencial recopilación de citas e ideas a su asunción y elaboración personal, de modo que se pueda realizar un trabajo original a partir de tal integración.
}

${ }^{28}$ En tal sentido, DE LORA y RUIZ MIGUEL (2018) apuntan que "en un trabajo de investigación se trata de dar razones en favor de la tesis-respuesta defendida a un problema previa y claramente acotado (..) No hay opiniones personales si por tal cosa entendemos juicios sin razones". Y es que, en todo caso, "los argumentos deben tener respaldo jurídico, es decir, el razonamiento no se hace en el vacío". Asimismo, y 
Y, en tercer lugar, se ha de insistir en que en la resolución del problema de que trate el TFG o en la elaboración del trabajo de investigación en marcha se ha de "llevar de la mano al lector". O dicho en otras palabras: el potencial destinatario ha de apreciar que existe un hilo conductor en la argumentación, de manera que sabe de dónde viene y hacia dónde se dirige, a la vez que puede identificar el camino o los medios a utilizar en tal recorrido. Este objetivo se consigue si el autor va haciendo introducciones del itinerario que va a seguir, de las razones para ello, o de los medios a utilizar. Igualmente, no está de más efectuar de vez en cuando algunas recapitulaciones para refrescar al lector la ruta de razonamiento que se sigue, así como avanzar lo que se ha de examinar, sin resolverlo anticipadamente ${ }^{29}$.

Finalmente, y a la vista de los resultados obtenidos, un consejo que considero de especial utilidad es que el estudiante deje "reposar" un tiempo el trabajo antes de entregárselo al tutor de manera que, tomando perspectiva, pueda re-enjuiciarlo él mismo antes de enviarlo a revisión. Esta lectura sosegada varios días o semanas después de su elaboración le permitirá detectar los propios fallos, ya formales, ya de falta de argumentación, ya de ausencia de hilo conductor. Cuando el estudiante ha interiorizado este modo de proceder y es quien se corrige a sí mismo, se puede decir que ha avanzado mucho en el objetivo de ser autónomo.

\section{Tarea tras la finalización del trabajo}

Una vez concluido el trabajo, solo resta al tutor dar algunas orientaciones al estudiante para la defensa del TFG. En este sentido, la experiencia fruto de este análisis muestra que no hay normas universales en cuánto al modo de exponer el trabajo, pero sí hay una

de manera conclusiva, señalan que un TFG viene a ser "una toma de postura razonada" sin que se convierta en una compilación, resumen o comentario de ideas ajenas.

${ }^{29}$ De manera similar se pronuncian DE LORA y RUIZ MIGUEL (2018) cuando afirman que "tiene que haber un hilo conductor y el lector tiene que saber desde el principio cuál es el camino que va a transitar: de dónde se parte, a dónde se quiere llegar y cómo". Para conseguir tal objetivo, recomiendan como buena fórmula "pensar en el lector y como si se estuviera en diálogo con el lector". A mi juicio, una consecuencia de tal modo de proceder es que la Introducción del TFG se escriba al final. En todo caso ha de ser clara y atractiva, pero no parece sencillo redactarla hasta que esté todo el trabajo culminado y, entonces, se pueda contar al lector el camino que en él se sigue. No resulta fácil delinear desde un principio el camino a recorrer, puesto que, en tal estadio, éste aun no se conoce en su totalidad. 
actitud que debe estar presente: "servir a la audiencia" ${ }^{3031}$. El modo que el estudiante escoja para hacer brillar todo lo posible el trabajo realizado ha de respetar ese principio si quiere llegar a buen puerto. Dos consecuencias inmediatas de asumir tal actitud son: por un lado, al pensarse más en la audiencia que en uno mismo, se disminuyen los nervios en la intervención -que habitualmente suelen deberse a que uno se centra más en su propia inseguridad-; y de otro, ayuda a enfocar el modo de exponer, sea cual sea la modalidad que se escoja -comentar las conclusiones, exponer una a una las preguntas, etc.-.

\section{Conclusiones}

Del estudio realizado a fin de resolver los problemas que usualmente manifiestan los estudiantes en la realización del TFG, y desde la perspectiva del tutor, de lo hasta ahora expuesto cabe entresacar, entre otras, tres conclusiones. La primera: el Trabajo de Fin de Grado constituye un hito importante en la formación del estudiante, no solo desde la perspectiva del currículo académico, sino, sobre todo, desde el punto de vista formativo. Por tal motivo parece oportuno cuidar de manera especial la labor tutelar que resulta necesaria para su desarrollo. Tal cuidado ha de manifestarse en la necesidad de formación específica para su desempeño, así como en el cultivo de habilidades de carácter interpersonal o relacional.

La segunda: los estudiantes agradecen que desde el principio se les haga ver el contenido de la relación tutelar. De manera específica, ha de transmitírseles que la iniciativa en la elaboración es suya, aun cuando cuenten con el apoyo y aliento constante del tutor. La tarea de este último, con una marcada vocación de servicio, ha de orientarse a proporcionar las herramientas necesarias para que los estudiantes adquieran autonomía en su aprendizaje. Lo que se persigue no es la elaboración de un buen trabajo en sí mismo considerado, sino que éste sea el resultado de la adquisición de habilidades y destrezas por parte de aquellos.

Y tercera: la experiencia demuestra que resulta muy útil diseñar detenidamente los pasos

o etapas en la tarea tutelar -reunión inicial, tipo de reuniones, etc.-, así como las

\footnotetext{
${ }^{30}$ Aludiendo a algunas pautas concretas de actuación para la estructura del discurso de defensa, así como para el uso del lenguaje -verbal y no verbal-, vid. SOLANO y MARTínEZ, 2012, 343-350.
}

31. 
herramientas a utilizar - guía tutelar, pautas de escritura, etc.- y comentarlas desde el inicio con los estudiantes.

\section{Bibliografía}

AltunA, A. (2005). El rol del orientador: tomas de posición para una orientación laboral más efectiva. Capital Humano, 192, 109-114.

BAIN, K. (2007). Lo que hacen los mejores profesores universitarios. Valencia: Publicaciones de la Universitat de València.

BAtTAner, E.; GonZÁlez, C. y SÁnchez, J.L. (2016). El Trabajo Fin de Grado (TFG) en las Universidades españolas. Análisis y discusión desde las defensorías universitarias. Rueda, 1, 44-82.

Beckman, M. y Hensel, N. (2009). Making explicit the implicit: Defining undergraduate research. CUR Quarterly, 29(4), 40-44.

Bonilla, M.I. y Martín, C. (2012). Evaluación de competencias en el Trabajo Fin de Grado en Administración y Dirección de Empresas: una propuesta de la Facultad de Ciencias Sociales de Talavera de la Reina (UCLM). Revista de Formación e Innovación educativa Universitaria, 5(4), 241-253.

CAnAbal, C. y Margalef, L. (2017). La retroalimentación: la clave para una evaluación orientada al aprendizaje. Revista de Currículum y Formación de Profesorado, 21(2), 149170.

CARlinO, P. (2002). Leer, escribir y aprender en la Universidad: ¿Cómo lo hacen en Australia y por qué? Investigaciones en Psicología, 7(2), 43-61.

DE LoRA, P. y RuIZ, A. (2018). ¿Cómo escribir un trabajo de investigación (TFG)? (en Derecho) en www.almacendederecho.org [última consulta 27/08/2020].

De Pro, A.; SÁnchez, G. y VAlcárcel, M.V. (2013). ¿En qué medida están contribuyendo los TFM a los resultados de aprendizaje planificados? Revista Eureka sobre Enseñanza y Divulgación de las Ciencias, 10, 728-748.

DíAZ-VÁzquez, R.; García-DíAZ, A.; Maside, J.M. y VÁzQueZ-Rozas, E. (2018). El trabajo de Fin de Grado: fines, modalidades y estilos de tutorización. Revista de Docencia Universitaria, 16(2), 159-175. 
García, N.; Asensio, I.; Carballo, R.; García, M. y Guardia, S. (2005). La tutoría universitaria ante el proceso de armonización europea. Revista de Educación, 337, 189210.

GonZÁLEZ, T. (2005). El Espacio Europeo de Educación Superior: una nueva oportunidad para la Universidad. En: P. Colas y J. de Pablos, La universidad en la Unión Europea. El espacio de la Educación Superior y su impacto en la docencia. Málaga: Aljibe (pp. 27$56)$.

Healey, M.; Lannin, L.; Stibbe, A. y Derounian, J. (2013). Developing and enhancing undergraduate final-year projects and dissertations. York, The Higher Education Academy. University of Gloucestershire.

HeInZE, A. y HeInZE, B. (2009). Blended e-learning skeleton of conversation: Improving formative assessment in undergraduate dissertation supervision. British Journal of Educational Technology, 40(2), 294-305.

Ingellis, A.G. y PICAZO, C. (2015). Análisis de un modelo de tutorización de TFG en el Espacio Europeo de Educación Superior: una aplicación en el Grado de Relaciones Laborales y Recursos Humanos. Lan Harremanak, 32 (I), 527-552.

MARín Vichis, R. (2017). Errores frecuentes en el uso de la metodología de la investigación jurídica. Revista de Pedagogía Universitaria y Didáctica del Derecho, 4(2), 87-98.

MARTín, B. (2016). La tutela del Trabajo de Fin de Grado: ¿cómo trabaja el profesorado? En: J. Gómez, E. López, L. Molina, A. Jaén y A.H. Martín (eds.), I Congreso Internacional en Formación, Investigación e Innovación Educativa. Libro de actas. San Juan, Puerto Rico (p. 149).

Martín, B. y Nieva, S. (2016). Herramientas para la tutela del Trabajo de fin de Grado. Una propuesta centrada en el y la estudiante. Universidad de Castilla-La Mancha.

Molina, Ma.D.; Rodríguez, J. y Colmenero, Ma.J. (2020). Importancia de la tutorización para el éxito del Trabajo de Fin de Grado. Revista Complutense de Educación, 31(2), 241-250.

Pozo, J.L. y MATEOS, M. (2009). Aprender a aprender: hacia una gestión autónoma y metacognitiva del aprendizaje. En: J.L. Pozo y M.P. Pérez (coords.), Psicología del 
aprendizaje universitario: la formación en competencias. Madrid: Ediciones Morata (pp. 54-69).

Río, Mª.L. del; DíAZ-VÁzQuez，R. y MAside, J.M. (2017). Satisfaction with the supervision of undergraduate dissertations. Active Learning in Higher Education, 19(2), 159-172.

Rodríguez, $\mathrm{M}^{\mathrm{a}}$.L. (coord.); Llanes, J. (coord.); Burguet, M.; BuXARRAIS, M ${ }^{\mathrm{a}}$. R.; Esteban, F.; Jarauta, B.; Molina, Ma .C.; Pérez, E.; Serrat, N. y Solé, M. (2013). Cómo elaborar, tutorizar y evaluar un Trabajo Fin de Máster. Agència per a la Qualitat del Sistema Universitari de Catalunya.

Rodríguez, S.; Álvarez, M.; Dorio, I.; Figuera, P.; FitA, E.; Forner, A. y Torrado, M. (2004). Manual de tutoría universitaria. Barcelona: Octaedro/ICE-UB.

Scardamalia, M. y Bereiter, C. (1992). Dos modelos explicativos de los procesos de composición escrita. Infancia y Aprendizaje, 58, 43-64.

Siota Álvarez, M. (2015). La tutoría entre iguales en el marco del Espacio Europeo de Educación Superior: fortalezas y requisitos necesarios para su implantación. Revista de Educación y Derecho, 11, 1-18.

SOLANO, I.M ${ }^{\mathrm{a}}$. y MARTínEZ, M${ }^{\mathrm{a}}$.J. (2012). Exposición oral y defensa del trabajo de fin de Grado y Máster. En: Mª.P. García y P. Martínez (coords.) (2012), Guía práctica para la realización de Trabajos Fin de Grado y Trabajos Fin de Máster. Murcia: Universidad de Murcia (pp. 315-350).

Tarí, J.J.; De Juana, J.; Valdés, J.; Andreu, R.; Manresa, E.; SAbater, V.; Rienda L. y FERNÁNDEZ, J.A. (2015). El proceso de elaboración y tutorización de los TFG y TFM a examen. En: J.D. Álvarez, Ma .T. Tortosa y N. Pellín (coords.), Investigación y Propuestas Innovadoras de Redes UA para la Mejora Docente. Alicante: Vicerrectorado de Estudios, Formación y Calidad, Instituto de Ciencias de la Educación (ICE), Universidad de Alicante (pp. 1357-1375).

TodD, M.; SMITH, K. y BANNISTER, P. (2006). Supervising a social science undergraduate dissertation: Staff experiences and perceptions. Teaching in Higher Education, 11(2), 161-173.

Tur-Viñes, V.; RodrígueZ, R.; Olivares, F.; Mora, F.J.; LÓPEZ-SÁNCHEZ, M.C.; Ramos, I.; Monserrat, J.; Campillo, C.; Segarra, J. y Borrás, M. (2013). 
Planificación y evaluación de la asignatura Trabajo Fin de Máster. En: J.D. Álvarez, $\mathrm{M}^{\mathrm{a}}$.T. Tortosa y N. Pellín (coords.), La producción científica y la actividad de innovación docente en proyectos de redes. Alicante: Vicerrectorado de Estudios, Formación y Calidad, Instituto de Ciencias de la Educación (ICE), Universidad de Alicante (pp. 86104).

VALCÁRCEl CASES, M. (coord.) (2003). La preparación del profesorado universitario español para la convergencia europea en Educación Superior. Dirección General de Universidades: programa de Estudio y Análisis.

Valderrama, E.; Rullán, M.; Sánchez, F.; Pons, J.; Mans, C.; Giné, F.; Seco, G.; Jiménez, L.; Peig, E.; Carrera, J.; Moreno, A.; García, J.; Pérez, J.; Vilanova, R.; Cores, F.; Renau, J.M.; Tejero, J. y Bisbal, J. (2010). La evaluación de competencias en los Trabajos Fin de Estudios. IEEE-RITA, 5(3), 107-114.

Vallés, C.; UreñA, N. y Ruiz, E. (2011). La Evaluación Formativa en Docencia Universitaria. Resultados globales de 41 estudios de caso. Revista de Docencia Universitaria, 9(1), 135-158.

WoOD, D.; BrunER, J.S. y Ross, G. (1976). The role of tutoring in problem solving. The Journal of Child Psychology and Psychiatry, 17, 89-100. 
Antonio Legerén Molina. Buenas prácticas en la dirección de trabajos de fin de grado. Especial referencia al

\begin{tabular}{|c|c|c|c|}
\hline $\begin{array}{l}\text { Anexo I } \\
\text { METACONOCIMIENTC }\end{array}$ & CONTENIDO & 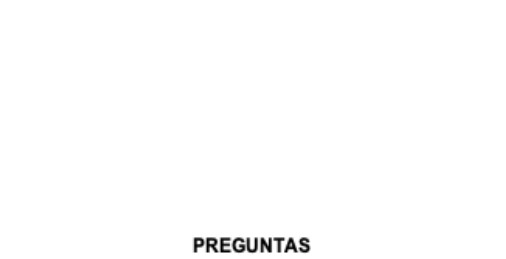 & HERRAMIENTAS \\
\hline \multirow{3}{*}{ Declarativo } & Conocer las propias competencias & $\begin{array}{c}\text { ¿Qué dificultades tengo para el TFG? } \\
\text { ¿Cuáles son mis habilidades? Investigación, bibliografia }\end{array}$ & Examen personal \\
\hline & Tareas a realizar & $\begin{array}{l}\text { ¿Cuál es el objetivo del TFG? } \\
\text { ¿En qué estado del trabajo estoy? }\end{array}$ & Contrastación con instrucciones TFG \\
\hline & Estrategias a usar & ¿Cómo proceder? & Lista de tareas \\
\hline \multirow{3}{*}{ Procedimental } & Planificación & ¿De qué tiempos dispongo? & Elaboración de calendario \\
\hline & Supervisión & ¿Es correcto mi trabajo? & $\begin{array}{l}\text { Supervisión en word } \\
\text { Tutoria personal }\end{array}$ \\
\hline & Evaluación & ¿Da el nivel mi TFG? & \\
\hline
\end{tabular}

